\title{
Aleitamento e parasitismo intestinal materno-infantil
}

\author{
Maternal and child intestinal parasitism and breast-feeding
}

\author{
Lêda Maria Costa-Macedo ${ }^{1}$ e Luis Rey ${ }^{2}$
}

\begin{abstract}
Resumo Entre março e maio de 1991, a prevalência das enteroparasitoses e o aleitamento materno foram determinados simultaneamente em 208 crianças menores de dois anos de idade e suas mães, atendidas em Instituição Pública de Saúde no Rio de Janeiro. Através da técnica de sedimentação, detectou-se positividade geral de 12,7\% para as crianças, e 37,3\% para as mães. Ascaris lumbricoides foi o parasito mais prevalente nas mulheres $(12,7 \%)$ e nos lactentes (4,3\%). A distribuição dos parasitos entre os grupos de aleitamento não variou para as mulheres, mas foi estatisticamente significativa em relação às crianças $(p<0,05)$. Nenhuma criança em aleitamento exclusivo apresentou parasitose. Verificou-se correlação positiva entre parasitismo e desmame. Cerca de 60\% das crianças parasitadas eram filhas de mães também parasitadas, sendo detectado um risco 1,7 vezes maior destas crianças virem a apresentar algum parasito intestinal. Acreditamos que a mãe parasitada possa influenciar na freqüência do parasitismo infantil.
\end{abstract}

Palavras-chaves: Parasitos intestinais. Aleitamento materno. Materno-infantil. Epidemiologia.

Abstract From March to May 1991, the prevalence of intestinal parasites was detected in 208 children under two-year old, from out patients attending the Public Health Service in Rio de Janeiro. Maternal parasitic infection was simultaneously investigated. Information regarding nurseling's breast-feeding and enteroparasites rates was also recorded. The centrifugalsedimentation was the method used for stool analysis. Positivity was observed in $12.7 \%$ for children and $37.3 \%$ for women. Ascaris lumbricoides was the most frequently detected parasite in children under one-year old (4.3\%) and mothers (12.7\%). The distribution of parasites accordingly to breast-feeding showed a significant statistical difference $(p<0.05)$ among infants. No exclusively suckled child presented infection. Children whose mothers were infected showed almost two fold risk of infection (1.7). A correlation between enteroparasites and weaned children was observed $(r=0.75)$. We believe that infected mothers can contribute for parasitic infection of their kids.

Key-words: Intestinal parasites. Breast-feeding. Infants. Epidemiology.

Contrastando com os avanços tecnológicos observados no fim do milênio, as parasitoses intestinais ainda se constituem um grande problema de saúde pública, sobretudo nos países em desenvolvimento, onde parasitos, como o Ascaris lumbricoides, são responsáveis por índices mais elevados de infecção ${ }^{1410}$.
A criança tem-se mostrado o alvo da infecção parasitária e é nela que as repercussões da parasitose tornam-se mais significativas ${ }^{521}$. No Brasil, mais da metade de pré-escolares e escolares encontra-se parasitada ${ }^{6}{ }^{18}$. Todavia, trabalhos sobre o parasitismo intestinal em crianças menores de dois anos são escassos

\footnotetext{
1. Disciplina de Parasitologia do Departamento de Patologia e Laboratórios da Universidade do Estado do Rio de Janeiro, Rio de Janeiro, RJ, Brasil. 2. Departamento de Medicina Tropical do Instituto Oswaldo Cruz/FIOCRUZ, Rio de Janeiro, RJ, Brasil.

Endereço para correspondência: Prof ${ }^{a}$ Lêda Maria da Costa Macedo. R. Manoel de Abreu 48/5ํandar, Vila Isabel, 20551-001 Rio de Janeiro, RJ, Brasil.

Telefax: 5521 587-6112

e-mail: leda@uerj.br

Recebido para publicação em 23/6/99.
} 
apesar de já se saber que a parasitose, sobretudo a ascariose, aparece cada vez mais precocemente nesta faixa etária ${ }^{691319}$.

Pouco se conhece sobre a interferência do aleitamento materno na prevalência do parasitismo intestinal. Porém, alguns autores detectaram associação entre aleitamento e prevalência diminuída de algumas parasitoses ${ }^{14}$.

A figura materna, preponderante nos primeiros anos de vida da criança, tem-se mostrado desassistida com relação ao controle dos enteroparasitos durante os períodos pré e pós-natal 2811161722 . Além disso, pouco se conhece sobre a influência do parasitismo materno na prevalência da infecção infantil, que é contestada por alguns autores ${ }^{11}$, porém admitida por outros ${ }^{322}$.

Os objetivos deste trabalho foram verificar a freqüência das enteroparasitoses em crianças menores de dois anos de idade, avaliar a simultaneidade do parasitismo intestinal materno e associar o parasitismo infantil com a forma de aleitamento natural realizado no momento do estudo.

\section{MATERIAL E MÉTODOS}

A amostra foi extraída do Serviço de Puericultura da Policlínica Piquet Carneiro (Ministério da Saúde/SUS/UERJ), localizado no Rio de Janeiro, entre março e maio de 1991.

A prevalência das enteroparasitoses foi verificada em 260 crianças menores de dois anos e em 220 mães, sendo que em 208 casos os exames foram realizados simultaneamente. Os potes para a coleta do material, uma amostra de fezes frescas de cada, foram distribuídos às mães após orientação.

O material foi processado pela técnica de sedimentação por centrifugação em sistema descartável (Parasitokit, Biotécnica), constituído de tubo cônico $(15 \mathrm{ml})$ e funil com extremidade porosa funcionando como filtro. O material foi diluído em água, filtrado e centrifugado a 1500 rotações por minuto, durante dois minutos.
Por entrevista, obtivemos informações sobre o aleitamento materno em 223 das crianças e 203 das mães incluídas no diagnóstico parasitológico, as quais foram distribuídas em grupos de aleitamento exclusivo, parcial e desmamadas.

As associações entre as variáveis relatadas no trabalho foram feitas utilizando-se o teste do qui-quadrado, com nível de significância de $95 \%$. Em uma das tabelas, foi calculada a razão de risco para avaliar as chances de uma criança, filha de mãe parasitada, de apresentar-se também parasitada, ou seja, consideramos o parasitismo materno como fator de risco ao parasitismo infantil. Foi utilizado o coeficiente de correlação de Pearson entre as variáveis parasitose infantil e desmame ${ }^{20}$.

\section{RESULTADOS}

A freqüência total dos parasitos encontrados nas crianças foi de $12,7 \%$, variando significativamente a positividade entre os grupos etários estudados $(p<0,05)$. Os parasitos mais frequentes foram A. lumbricoides para os menores de um ano e Giardia lamblia para os de um ano de idade (Figura 1). Entre as crianças de um ano, dois exames foram positivos para Entamoeba coli e um para Endolimax nana. Somente nesta faixa etária foi evidenciado poliparasitismo em $3(1,1 \%)$ crianças.

O parasitismo materno esteve presente em $37,3 \%$ das mulheres $(n=220)$, sendo liderado pelo $A$. lumbricoides, responsável por $12,7 \%$ das infecções parasitárias, seguido por G. lamblia com $5 \%$ e Trichuris trichiura com 3,6\%. A distribuição das mulheres nos grupos, segundo o aleitamento materno, mostrou que a distribuição dos parasitos não variou conforme os grupos. Para as crianças, no entanto, a associação entre parasitismo e aleitamento foi verificada estatisticamente. Nenhum caso de parasitismo foi observado nas 32 crianças em aleitamento exclusivo (Tabela 1).

O perfil de aleitamento materno, segundo a idade das crianças estudadas, é apresentado na Figura 2. Observa-se que o aleitamento exclusivo é realizado em mais de $70 \%$ dos lactentes menores de três meses, mas em apenas 17\% após esta idade. Após os seis meses, mais da metade das crianças já foi desmamada. Neste gráfico, as linhas que representam as freqüências das crianças parasitadas e a de desmamadas variaram num mesmo sentido indicando uma correlação positiva entre estas variáveis $(r=0,75)$.

Com relação à simultaneidade do parasitismo materno-infantil, a Tabela 2 mostra que quase 
$60 \%$ das crianças parasitadas eram filhas de mães também infectadas. Houve associação estatisticamente significativa entre o parasitismo materno e o infantil $(p<0,05)$, detectando-se um risco 1,7 vezes maior para a criança, filha de mãe parasitada, de adquirir a infecção.

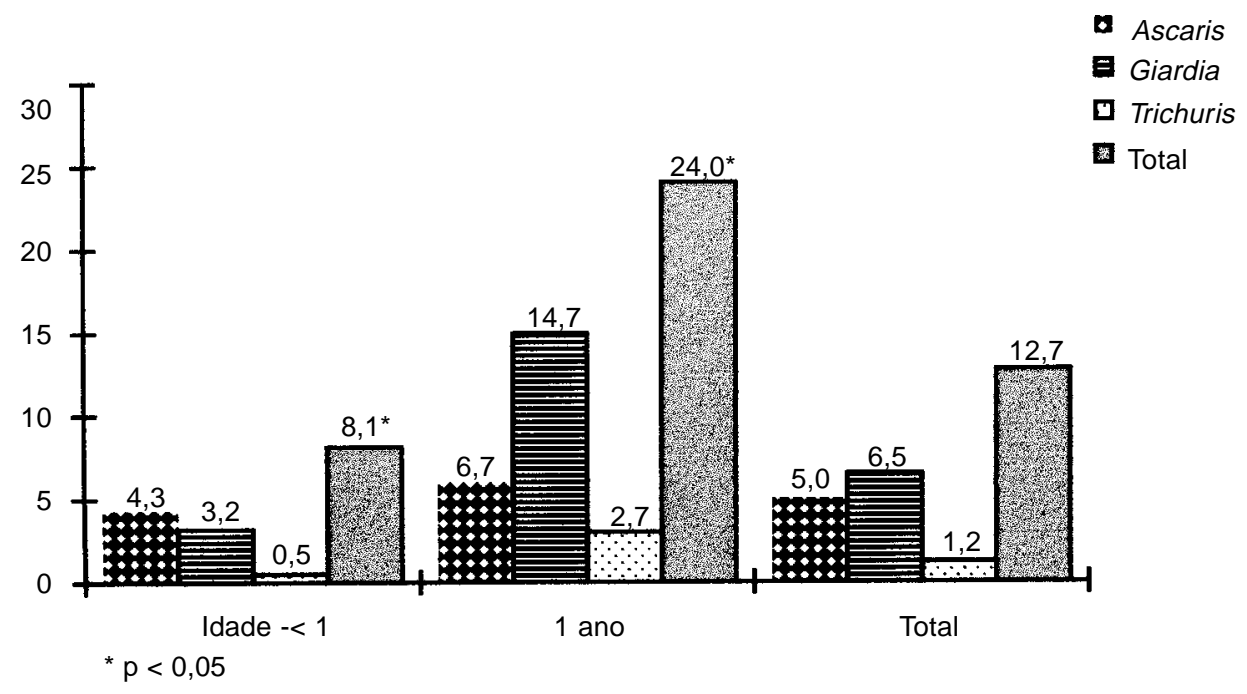

Figura 1 - Freqüência dos parasitos patogênicos encontrados segundo a idade das crianças.

Tabela 1 - Distribuição do parasitismo intestinal materno e infantil segundo o tipo de aleitamento.

\begin{tabular}{|c|c|c|c|c|c|c|}
\hline \multirow{3}{*}{ Aleitamento } & \multicolumn{3}{|c|}{ Mães } & \multicolumn{3}{|c|}{ Crianças } \\
\hline & \multirow[t]{2}{*}{ examinadas } & \multicolumn{2}{|c|}{ positivas * } & \multirow[t]{2}{*}{ examinadas } & \multicolumn{2}{|c|}{ positivas ** } \\
\hline & & $\mathrm{n}^{\circ}$ & $\%$ & & $\mathrm{n}^{\circ}$ & $\%$ \\
\hline Exclusivo & 29 & 12 & 41,4 & 32 & - & \\
\hline Parcial & 71 & 27 & 38,0 & 75 & 8 & 10,7 \\
\hline Não aleitadas & 103 & 39 & 37,9 & 116 & 20 & 17,2 \\
\hline Total & 203 & 78 & & 223 & 28 & \\
\hline
\end{tabular}

* não significativo ** $p<0,05$

Tabela 2 - Simultaneidade de parasitismo intestinal entre mãe e filho.

\begin{tabular}{|c|c|c|c|c|c|}
\hline \multirow{3}{*}{ Mãe } & \multicolumn{4}{|c|}{ Criança } & \multirow{3}{*}{$\begin{array}{c}\text { Total } \\
\text { no }\end{array}$} \\
\hline & \multicolumn{2}{|c|}{ positiva * } & \multicolumn{2}{|c|}{ negativa } & \\
\hline & $\mathrm{n}^{\circ}$ & $\%$ & $\mathrm{n}^{\circ}$ & $\%$ & \\
\hline Positiva & 13 & 59,1 & 63 & 33,9 & 76 \\
\hline Negativa & 9 & 40,9 & 123 & 66,1 & 132 \\
\hline Total & 22 & 100 & 186 & 100 & 208 \\
\hline
\end{tabular}

${ }^{*} p<0,05$ razão de risco: 1,7 


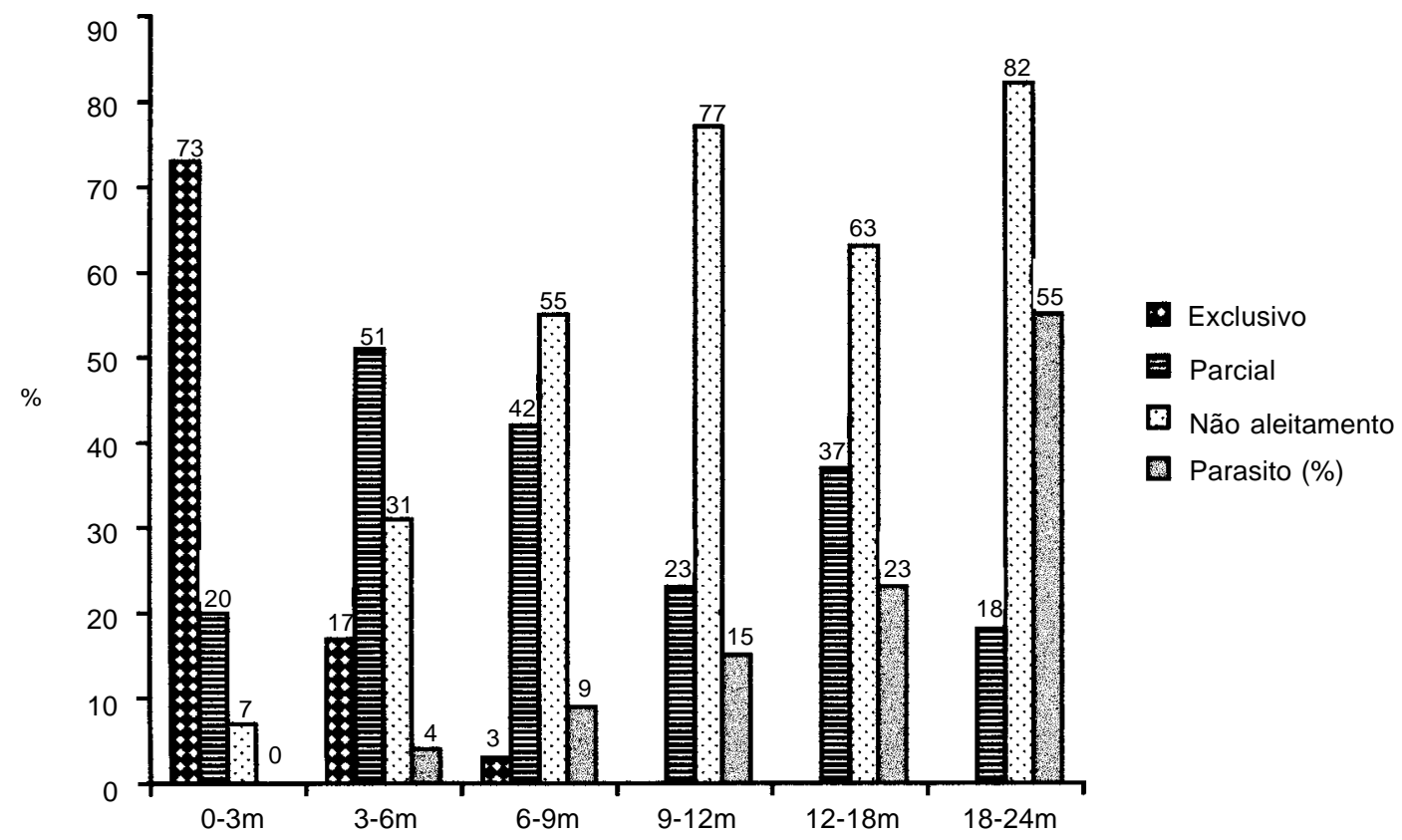

Figura 2 - Distribuição das crianças estudadas segundo o tipo de aleitamento materno efetuado e o parasitismo intestinal, de acordo com a idade (meses).

\section{DISCUSSÃO}

As parasitoses intestinais, apesar de bem conhecidas nos países em desenvolvimento, são pouco estudadas no segmento materno-infantil da população. Além do desconhecimento das freqüências de parasitismo nestes indivíduos, as drogas antiparasitárias, por serem pouco estudadas quanto às repercussões em gestantes, puérperas e em crianças menores de dois anos, são pouco recomendadas a esta população.

Com relação à prevalência das enteroparasitoses nas crianças, observou-se infecção em $24 \%$ das crianças de um ano de idade, percentual significativo dado a capacidade espoliativa do parasito mais encontrado nesta faixa etária ( $G$. lamblia). O percentual de ascariose encontrado entre os lactentes confirma 0 achado em outros trabalhos ${ }^{6}$ e nos surpreende pelo fato dessa geohelmintose, com período prépatente em torno de dois meses, infectar tão precocemente essas crianças.

O perfil de aleitamento das crianças encontrado neste estudo (Figura 2) concorda com o apresentado pelo IBGE (1992), onde constata-se que em crianças de baixa renda 0 desmame é precoce. Têm-se estudado a importância do leite materno na diminuição do parasitismo infantil. Em estudos in vitro com o leite humano, foi identificada enzima com atividades giardicida ${ }^{15}$. Outros autores detectaram taxas menores de parasitismo por $A$. lumbricoides e G. lamblia em lactentes aleitados naturalmente ${ }^{14}$. A correlação positiva entre o desmame e o parasitismo infantil (Figura 2), leva-nos a sugerir que medidas de incentivo ao aleitamento natural sejam instituídas como contribuição ao controle das enteroparasitoses nos lactentes.

As taxas de parasitismo materno distribuídas homogeneamente entre os grupos de aleitamento estabelecidos neste estudo, sugerem que as crianças e as mães estavam igualmente expostas ao risco de infecção parasitária. Entretanto, nenhuma criança apresentou-se infectada quando em aleitamento exclusivo, verificandose associação estatística entre aleitamento materno e a freqüência do parasitismo infantil (Tabela 1).

A simultaneidade encontrada entre o parasitismo materno e o infantil mostrou um risco 1,7 vezes maior para o filho da mulher parasitada de apresentar também infecção parasitária (Tabela 2). Alguns trabalhos ressaltam a importância da contaminação ambiental facilitada 
por manipuladores de alimentos, estreitamente relacionados ao lactente ${ }^{12}$, ou da própria mãe ${ }^{13}$, influenciando na prevalência das parasitoses intestinais dos lactentes. Outros recorrem a mecanismos imunológicos para explicar o baixo parasitismo dos lactentes seguido por súbita elevação nas crianças de um ano, considerando que a passagem de anticorpos maternos, de mãe imune, protegeria a prole nos primeiros meses, porém tornando-a mais susceptível após ${ }^{3}$.

O método realizado já foi verificado ser de baixa sensibilidade, com percentual considerável de falsos-negativos ${ }^{17}$. Desta forma, o parasitismo

\section{REFERÊNCIAS}

1. Bundy DAP. This wormy world-then and now. Parasitology Today 13:407-408, 1997.

2. Caceres MR, Costa-Macedo LM. Controle de enteroparasitoses em puérperas do Município do Rio de Janeiro. Jornal Brasileiro de Medicina 73:45-49, 1997.

3. Carlier Y, Truyens C. Influence of maternal infections on offspring resistence towards parasites. Parasitology Today 11:94-99, 1995.

4. Chan MS. The global burden of intestinal nematode infections-fifty years on. Parasitology Today 13:438-443, 1997.

5. Chan MS, Medley GF, Jamison D, Bundy DAP. The evaluation of potential global morbity attributable to intestinal nematode infections. Parasitology 109:373-387, 1994.

6. Costa-Macedo LM, Costa MCE, Almeida LM. Parasitismo pelo Ascaris lumbricoides em crianças menores de dois anos em comunidade aberta do Rio de Janeiro. Cadernos de Saúde Pública 15:173-178, 1999.

7. Costa-Macedo LM, Machado-Silva JR, Rodrigues-Silva R, Oliveira LM, Vianna MSR. Enteroparasitoses em préescolares de comunidades favelizadas da cidade do Rio de Janeiro, Brasil. Cadernos de Saúde Pública 14:851 855, 1998.

8. Costa-Macedo LM, Rey L. Enteroparasitoses em gestantes e puérperas no Rio de Janeiro. Cadernos de Saúde Pública 12:383-388, 1996.

9. Costa-Macedo LM, Rey L. Frequency e precocity of human intestinal parasitism in a group of infants from Rio de Janeiro, Brasil. Revista do Instituto de Medicina Tropical de São Paulo 39:305-306, 1997.

10. Crompton DWT, Savioli L. Intestinal parasitic infections and urbanization. Bulletin of the World Health Organization 71:1-7, 1993

11. D'Alauro F, Lee RV, Pao-in K, Khairallah M. Intestinal parasites and pregnancy. Obstetrics and Gynecology 66:639-643, 1985

12. Frenzel A, Torres $P$, Guerrero $S$, Gesche W, Montefusco A, Marin F. Parasitosis intestinal en lactentes y su relación con la infección de sus manipuladores de alimentos y el saneamiento ambiental. Revista Médica de Chile 107:343-351, 1979. intestinal encontrado entre mãe e filho pode apresentar-se subestimado neste trabalho.

Diante desses achados, acreditamos que a mãe parasitada possa influenciar na freqüência do parasitismo infantil seja diretamente, como no caso de transmissão das protozooses intestinais; seja indiretamente, contribuindo para o aumento da contaminação ambiental; seja como intermediária, veiculando parasitos geohelmintos, como no caso do A. lumbricoides, do meio contaminado (peridomiciliar) para a criança (intradomiciliar).

\section{BIBLIOGRÁFICAS}

13. Gendrel D, Kombila M, Khayati A, Bourdil M, Engohan E, Richard-Lenoble D. Précocité d'apparition du parasitisme intestinal chez le nourisson en Afrique Equatoriale. In: Annales de Pédiatrie 30:453456, 1983.

14. Gendrel D, Richard-Lenoble D, Kombila M, Baziomo JM, Gendrel C, Nardou M. Allaitement maternel et parasites intestinaux. Archives Françaises de Pédiatrie 45:399-404, 1988.

15. Gillin FD, Reiner DS. Human milk kills parasitic intestinal protozoa. Science 221:1290-1292, 1983.

16. Guerra EM, Vaz AJ, Toledo LAS, Lanoni AS, Quadros CMS, Dias RMDS, Barreto OCO. Infecções por helmintos e protozoários intestinais em gestantes de primeira consulta atendidos em centros de saúde da rede estadual no Subdistrito do Butantã, Município de São Paulo. Revista do Instituto de Medicina Tropical de São Paulo 33:303-308, 1991

17. Machado FHS, Costa-Macedo LM. Prevalência de enteroparasitoses em gestantes de baixa renda do município do Rio de Janeiro. Jornal Brasileiro de Patologia 32:20-25, 1996.

18. Monteiro CA, Chieffi PP, Benício MHA, Dias RMS, Torres DMAGV, Mangini ACS. Estudo das condições de saúde das crianças do Município de São Paulo (Brasil), 1984/ 1985. VII. Parasitoses intestinais. Revista de Saúde Pública 22:8-15, 1988.

19. Santana LR, Alencar MJM, Rouquayrol MZ. Poliparasitismo intestinal e recidiva de enteroparasitoses em crianças de tenra idade. Revista Brasileira de Análises Clínicas 26: 50-52, 1994

20. Siegel S. Estatística não paramétrica. McGraw Hill, São Paulo, 1975.

21. Silva NR, Chan MS, Bundy DAP. Morbidity and mortality due to ascariasis: re-estimation and sensivity analysis of global numbers at risk. Tropical Medicine International Health 2:519-528, 1997.

22. Villar J, Klebanoff M, Kestler E. The effect on fetal growth of protozoan and helminthic infection during pregnancy. Obstetrics and Gynecology 74:915-920, 1989. 